\title{
Inhibiting Effects of Myrmicacin and Homologous Compounds on Cleavage of Hemicentrotus pulcherrimus Eggs
}

\author{
Yozo Iwanami, Eigoro Tazawa and Tsukasa Iwadare* \\ Biological Institute, Yokohama City University, Kanazawa-Ku, Yokohama 236 \\ and $*$ Research Laboratory, Sakura Finetechnical Co. Ltd., Tokyo 103, Japan
}

\begin{abstract}
Carboxylic acids containing 7 carbons or more such as enanthic, caprylic, pelargonic and $\beta$-hydroxydecanoic acids inhibited cleavage of Hemicentrotus pulcherrimus eggs at a concentration of $200 \mathrm{ppm}$. Decan-3-o1, in which the carboxyl group is replaced with the methyl group, had no inhibiting effect. $\beta$-hydroxydecanoic acid (myrmicacin) had a strong inhibiting effect that was almost completely reversed by transfer of the eggs to a medium lacking the acid after a limited period of exposure.
\end{abstract}

Myrmicacin, which occurs in secretions of the South American leaf-cutting ant (Atta sexdens), was first isolated and identified as $\beta$-hydroxydecanoic acid by Schildknecht and Koob in 1971 (6). The compound was assumed to prevent germination of the ants' harvests, and it has been synthesized by various methods $(5,7)$. Schildknecht and Koob (6) observed that myrmicacin inhibited germination of spores of Altenaria and Botrytis, and Iwanami and Iwadare (1) reported that the agent inhibited pollen growth and pollen tube mitosis in several higher plants. It also has been observed that myrmicacin stopped the mitotic progression of Ornithogalum pollen at all stages, even after metaphase (2), and that some fatty acids (for which the group name "Myrmic acids" has been proposed (3) ) stopped pollen germination, pollen tube elongation, and mitotic division of generative nucleus, as did myrmicacin.

These recent results led us to test the effects of myrmicacin and its homologs on the cell division of animals. We confirmed that these chemicals inhibit the cleavage of fertilized eggs of a sea urchin. The results of our study are described here.

The material used was the sea urchin, Hemicentrotus pulcherrimus, collected from Sagami Bay, Kanagawa Prefecture, Japan and stored in aerated sea water tank before use. The spawning of sea urchin gametes was stimulated by pouring $0.5 \mathrm{M}$ potassium chloride solution into the body cavity after excision of the oral region. The eggs were washed with filtered sea water, then suspended in the sea water at an apporoximate concentration for fertilization. A sample was taken to be used as the suspension of unfertilized eggs. A seprm suspension adequate to insure a $95 \%$ or more incidence of fertilization was added to the egg suspension. Fig. 1-1 shows the process and time scale of fertilization and cleavage of the eggs of Hemicentrotus pulcherrimus at $20-22^{\circ} \mathrm{C}$. The chemicals used are listed in Fig. 1-2. They included five carboxylic acids containing 6-10 carbons and an alcohol (decan-3-o1), in which the carboxyl group in myrmicacin is replaced with a methyl group. Myrmicacin was synthesized as dl- $\beta$-hydroxydecanoic acid by Meyers' method (5). Other compounds were purchased from Tokyo Kasei Co. Ltd. 

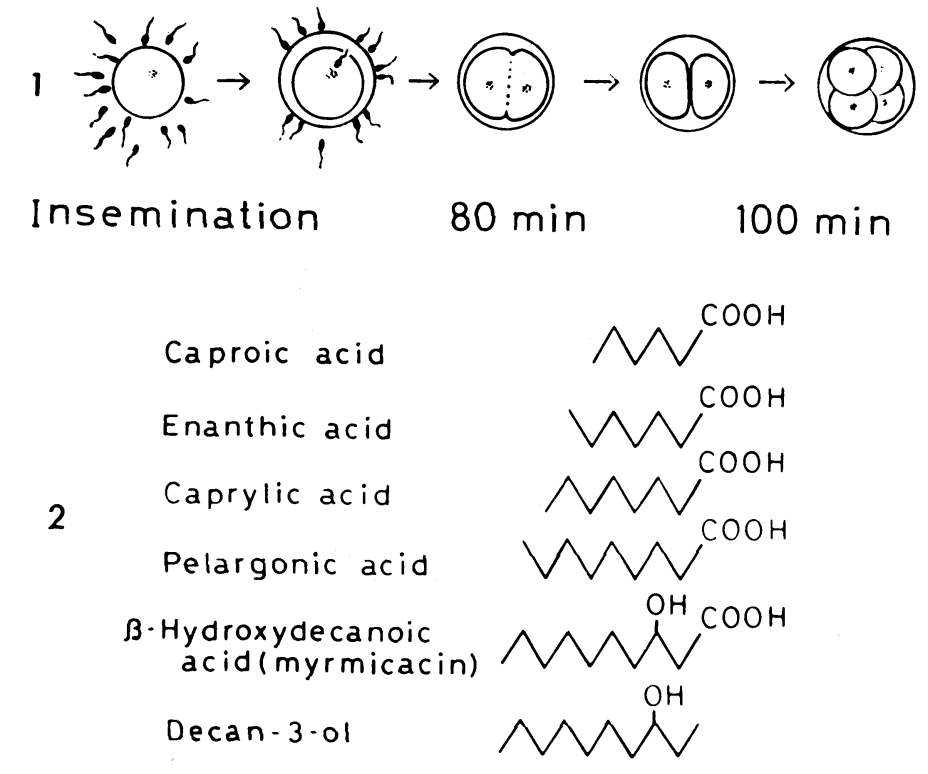

Fig. 1. Schematic diagram and time scale of the cleavage of the eggs of sea urchin, Hemicentrotus pulcherrimus at $22^{\circ} \mathrm{C}(1)$ and the chemical structures of the 6 compounds (2) used in this study.

The effects of the compounds on cleavage of the fertilized eggs of the sea urchin was investigated in solution of each of the compounds $(200 \mathrm{ppm})$ in sea water. The $\mathrm{pHs}$ of the solutions of compounds in sea water $(\mathrm{pH} 8.2)$ were below 7.5, and were not adjusted to 8.2 because the inhibiting effect of myrmicacin decreases under alkaline conditions $(2,3)$ and because the biological effect of the compound used to adjust the $\mathrm{pH}$ had to be considered. Therefore, two controls (A, B) were prepared and the $\mathrm{pH}$ of one (A) was adjusted to 7.5 by titration with diluted acetic acid.

The solution containing the compounds, and the controls were placed in separate Petri-dishes $3 \mathrm{~cm}$ in diameter. Approximately 2,000 eggs were suspended in each solution, and the sperm suspension was added about 20 min after suspension of the eggs. To observe cleavage, samples containing about 200 eggs were pipetted out at regular intervals and placed on slide glasses, then the number of eggs cleaved into two or more cells was counted under a microscope. Inhibited eggs were washed twice with filtered sea water, then kept in the normal sea water (agent-free). Experiments were repeated three times, and the mean values were determined.

In a preliminary experiment, the agents had no effect on the biological activity of sperm because inseminated eggs treated with these compounds for 20 min cleaved normally. The eggs treated with all the agents, except caproic and enanthic acid, raised normal fertilization membranes. Approximately half of the eggs raised the membrane when treated with caproic or enanthic acid, and cleavage was observed only in eggs with the membrane. The effects of the agents on the cleavage of fertilized eggs are shown at the top of Fig. 2.

In normal sea water (A) or in $\mathrm{pH}$-adjusted sea water (B), about $98 \%$ of the eggs cleaved into two or more cells within $1.5 \mathrm{~h}$ after insemination, but the rate of cleavage of eggs treated with caproic acid or decan-3-ol was somewhat lower than that of the control, and a number of abnormal cleavages was observed. In sea water containing 


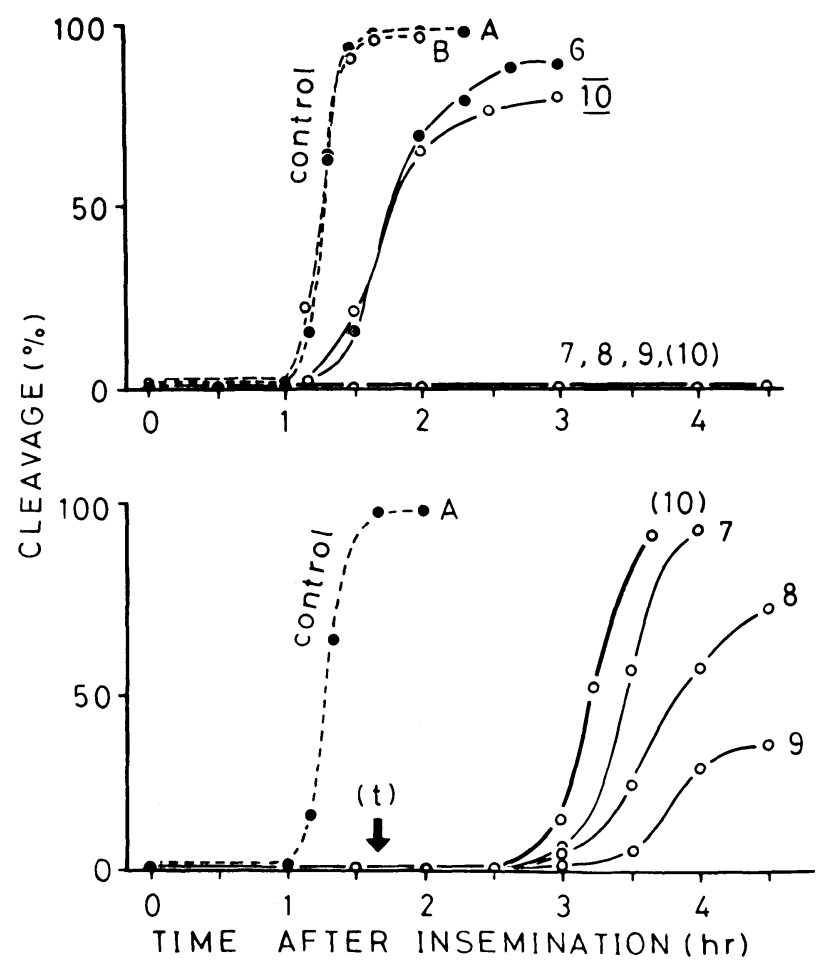

Fig. 2. Comparison of the effects of 6 chemicals on cleavage of sea urchin eggs (top) and removal of inhibition when the eggs were transferred to normal sea water (bottom). control, normal sea water (A, pH 8.2; B, pH 7.5); 6, caproic acid; 7, enanthic acid; 8, caprylic acid; 9, pelargonic acid; (10), myrmicacin; 10 , decan-3-o1; Numbers $6-10$ are the carbon atom number of 6 chemicals. $(\mathrm{t})$, treated eggs were transferred to normal sea water.

enanthic, caprylic, or pelargonic acid, or myrmicacin, no cleavage took place even after $4 \mathrm{~h}$. Also, noticeable deformation of the eggs accompanied by shrinkage occurred. These results are fairly similar to the inhibiting effects of the agents on pollen growth and pollen tube mitosis in that a carboxyl group and 8-10 carbons were essential for inhibition (3).

To check whether this inhibition can be removed, eggs treated with enanthic, caprylic, pelargonic acids or myrmicacin ( $\beta$-hydroxydecanoic acid) were transferred to normal sea water 100 min after insemination, and the number of cleaved eggs was counted. The results are shown at the bottom of Fig. 2. The eggs, whose cleavage had been arrested by one of these agents for $100 \mathrm{~min}$, gradually resumed normal shape when transferred to agent-free sea water, and cleavage took place after $60-90 \mathrm{~min}$ of transfer. Eggs treated with the higher acids had less cleavage restoration as shown in Fig. 2, but myrmicacin, in spite of its 10 carbons, showed a restoration comparable to that of enanthic acid. These results are similar to those for pollen growth and pollen tube mitosis (3). Arresting cleavage for longer than $2 \mathrm{~h}$, and the subsequent transfer to agent-free sea water induced abnormal cleavage in some eggs.

The mechanism of inhibition by these agents is not known. However, since it took $1.5 \mathrm{~h}$ for the eggs to begin to cleave after transfer from the inhibitor-containing 
medium to the inhibitor-free medium in this experiment (Fig. 2), and since Iwanami has observed that mitotic division of the generative nucleus of Ornithogalum pollen was stopped at all stage, even after metaphase, by myrmicacin (2), we assume that inhibition of cell division in the sea urchin may take place at various stages. A detailed study on this subject is in progress. We also have confirmed that the propagation of HeLa cells temporarily was inhibited in a medium containing $125 \mathrm{ppm}$ myrmicacin (4).

Thus, the agents called "myrmic acids (MYA)" (3) may be used as biological inhibitors for various studies. Myrmicacin, from the secretions of ants, is the best of these agents, because its inhibiting activity is strong; it is not toxic (over a short term) to cells; and its effects are eliminated when the agent is removed from the cells.

\section{REFERENCES}

1. Iwanami, Y. and T. Iwadare. Inhibiting effects of myrmicacin on pollen growth and pollen tube mitosis. Bot. Gaz. 139, 42-45, 1978

2. IwANAmi, Y. Myrmicacin, a new inhibitor for mitotic progression after metaphase. Protoplasma 96, 267-271, 1978

3. IWANAmi, Y. and T. IWADARE. Myrmic acids; a group of new inhibitors analogous to myrmicacin ( $\beta$-hydroxydecanoic acid). Bot. Gaz. (in press)

4. Iwanami, Y., H. FukuYasu, K. Kawakami and Y. Kazuno. Inhibitory effect of myrmicacin ( $\beta$-hydroxydecanoic acid) on propagation of HeLa cells. (in preparation)

5. Meyers, A.I. and D.L. Temple Jr. Syntheses via 2-oxazolines. II. A versatile synthesis of aliphatic carboxylic acids and esters. Mono- and dialkylation of acids masked by a simple protecting group. J. Amer. Chem. Soc. 92, 6644-6646, 1970

6. Schild necht, H. and K. Коов. The first insect herbicide. Angew. Chem. Int. Edit. 10, 124125,1971

7. SKoGH, M. The higher normal chain DL- $\beta$-hydroxy acids: Synthesis and investigation of the crystal behaviour of seventeen homologous acids with 8-24 carbon atoms. Acta. Chem. Scand. 6, 809-817, 1952 